\title{
Supplemental Material for Stress-strain engineering of single-crystalline silicon membranes by ion implantation: Towards direct-gap group-IV semiconductors
}

\author{
Mateus G. Masteghin, ${ }^{1,2}$ Vivian Tong, ${ }^{3}$ Eleanor B. Schneider, ${ }^{1,2}$ Cameron C. L. Underwood, ${ }^{1}$ \\ Tomas Peach, ${ }^{1}$ Benedict N. Murdin, ${ }^{1}$ Roger P. Webb, ${ }^{1,2}$ Steven K. Clowes, ${ }^{1}$ and \\ David C. Cox ${ }^{1,2,3, *}$ \\ ${ }^{1}$ Advanced Technology Institute, University of Surrey, Guildford, GU2 7XH, UK. \\ ${ }^{2}$ Ion Beam Centre, University of Surrey, Guildford, GU2 7XH, UK. \\ ${ }^{3}$ National Physical Laboratory, Teddington, TW11 0LW, UK.
}

\section{FLATTENING A BUCKLED SILICON MEMBRANE}

In the same way that a germanium $(\mathrm{Ge})$ layer deposited onto silicon $(\mathrm{Si})$ generates strain at the $\mathrm{Ge} / \mathrm{Si}$ interface, a silicon dioxide $\left(\mathrm{SiO}_{2}\right)$ layer also strains the $\mathrm{Si} / \mathrm{SiO}_{2}$ boundary due to expansions undergone during the oxidation, with different thermal expansion coefficients [1-3]. However, during the back etch of a silicon-on-insulator (SOI) substrate to create free-standing Si membranes, the strained $\mathrm{Si}$ atoms equilibrate causes buckling (reference Fig. 2(a) of main manuscript). This buckling prevents the widespread use of these single-crystal Si membranes as TEM grids. Moreover, a wrinkled membrane prevents future device processing such as lithographic techniques.

In order to better understand the dosing effect on the flattening process of a membrane by $\mathrm{Xe}^{+}$ ions implantations, a detailed evolution of wrinkles is depicted in the Supporting Animation (GIF). To produce the GIF, a $20 \mu \mathrm{m}$ width annulus was exposed in steps of 2 seconds using a current of about 150 pA (dose increments of $1.17 \times 10^{13}$ ions $\mathrm{cm}^{-2} \mathrm{~s}^{-1}$ ). Using a scanning transmission electron microscopy (STEM) detector in the bright field mode, wrinkles and bend contours can be easily detected when operating the SEM at $30 \mathrm{keV}$. From the GIF we concluded that a course flattening is observed at approximately $10^{14}$ ions $\mathrm{cm}^{-2}$, i.e. a quarter of the full amorphization dose obtained from SUSPRE. This corresponds to the dose in which half of the host atoms that have been displaced by implanted ions, as shown in Fig. 3. Increasing the dose above this point reduced the wrinkles formed on the edge of the amorphous part, but no further significant changes are noticeable at the center of the membrane (Fig. 1). This illustrates how the ion implants gently induce the contraction/ bending of the exposed area. Note 
that during the implantation only the top half of the tensioner thickness undergoes amorphization, being crucial to the bi-material bending mechanism discussed in the main text.

The diffraction patterns in Fig. 2(d-f) of main manuscript and Fig. 2 were obtained using the STEM detector in the high angle dark field (HADF) mode, and the electron beam in the channelling mode. In the channelling mode, a parallel beam rocks periodically around a point at the sample surface, and the diffracted signal is used to create Kikuchi bands [4]. From analysis of the diffraction patterns of Fig. 2, it is found that the angle between the planes (400) and (040) approaches $90^{\circ}$ only after exposing the annulus to the full amorphization dose. When the exposed dose is $1.00 \times 10^{14}$ ions $\mathrm{cm}^{-2}$, the angle is $86.8^{\circ}$, reaching $89.4^{\circ}$ with $1.75 \times 10^{14}$ ions $\mathrm{cm}^{-2}$, and finally $90^{\circ}$ at $3.75 \times 10^{14}$ ions $\mathrm{cm}^{-2}$. We expect these angles to differ from $90^{\circ}$ as a result of elastic deformation in order to accommodate the buckling.

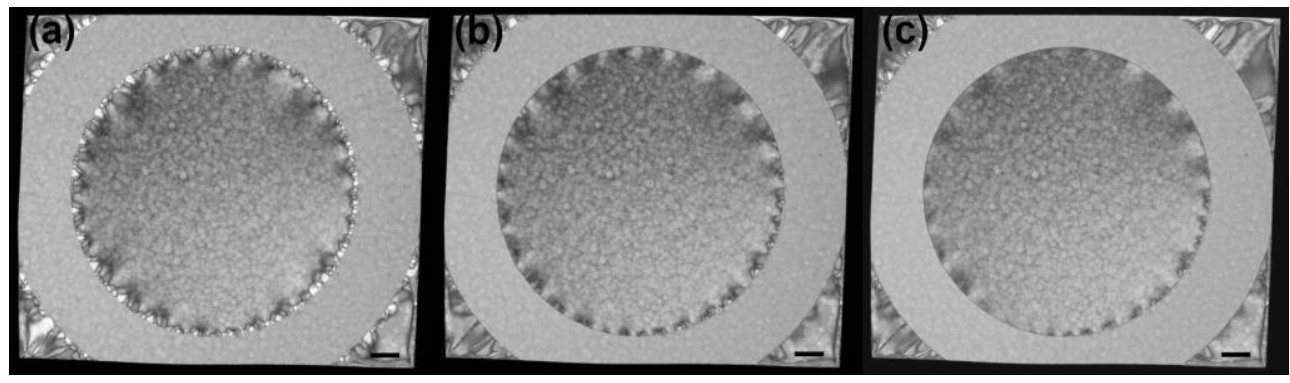

FIG. 1. As main manuscript Fig. 2 (a-c) for another silicon membrane exposed to a $30 \mathrm{keV} \mathrm{Xe}^{+}$beam with (a) $3.75 \times 10^{14}$ ions $\mathrm{cm}^{-2}$ ), (b) $7.50 \times 10^{14}$ ions $\mathrm{cm}^{-2}$, and (c) $11.25 \times 10^{14}$ ions $\mathrm{cm}^{-2}$. This onset for amorphization of $3.75 \times 10^{14}$ ions $\mathrm{cm}^{-2}$ was obtained using SUSPRE. Scale bars correspond to $10 \mu \mathrm{m}$.

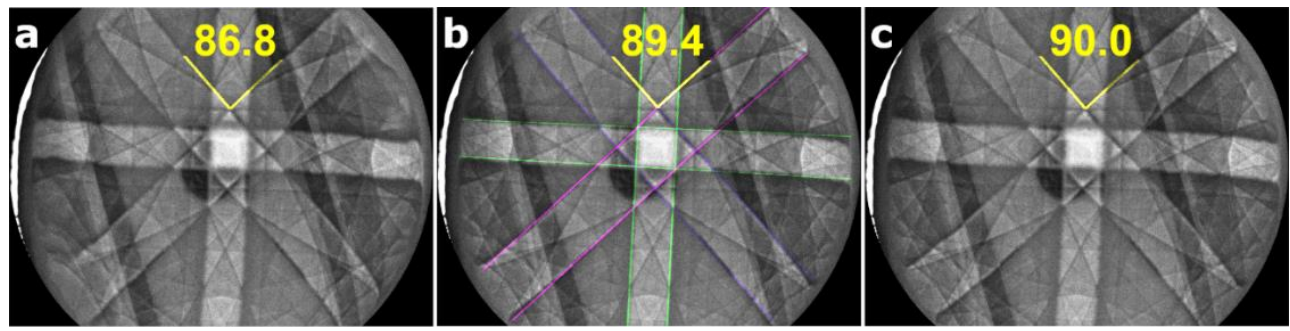

FIG. 2. As main manuscript Fig. 2 (d-f) for another silicon membrane exposed to a $30 \mathrm{keV} \mathrm{Xe}$ beam. Diffraction patterns obtained using the HADF-STEM detector for exposure patterns with the same geometry of Fig. 1, but doses of (a) $1.00 \times 10^{14}$ ions $\mathrm{cm}^{-2}$, (b) $1.87 \times 10^{14}$ ions $\mathrm{cm}^{-2}$, and (c) $3.75 \times 10^{14}$ ions $\mathrm{cm}^{-2}$. The angle between planes |400| (purple contours) and |040| (magenta contours) only reached the expected $90^{\circ}$ with the annulus exposed to the full amorphization dose. The green contours highlight the $|220|$ planes and the Kikuchi lines are the ones expected for a (100) silicon single crystal. The maximum angle of the DP is $10.8^{\circ}$. 


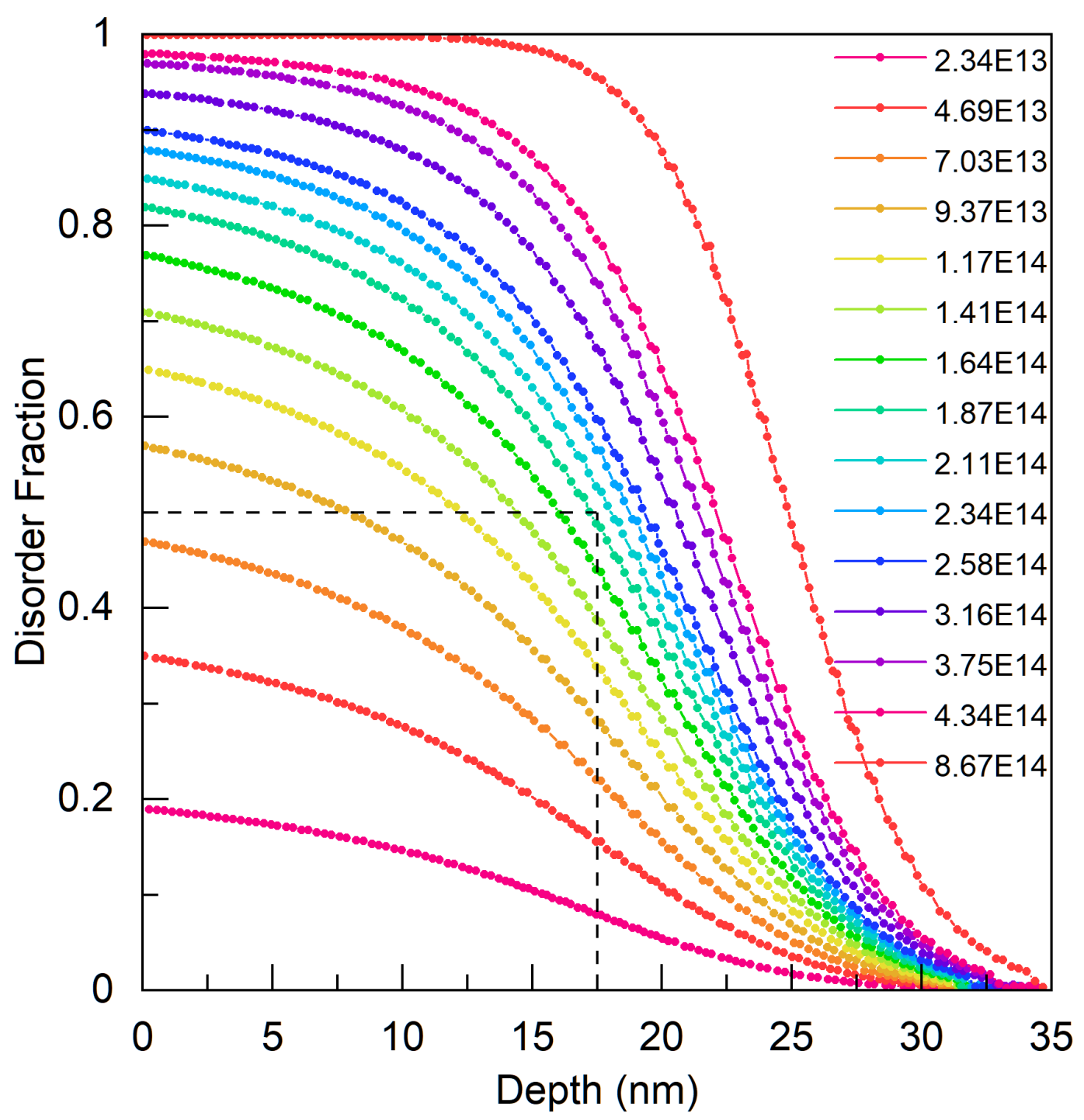

FIG. 3. Supporting image for the animation GIF showing the flattening of a wrinkled membrane. This plot alongside the supporting animation shows that a dose of $1.87 \times 10^{14}$ ion $\mathrm{cm}^{-2}$ is enough to turn half of the silicon membrane into an amorphous layer, endorsing the bilayer mechanism of strain generation using ion beam implantation based on the wrinkle annihilation in the supporting animation. The ordinate shows the fraction of host atoms that have been displaced by implant ions calculated using the SUSPRE model. 


\section{BIAXIAL STRAIN ENGINEERING}

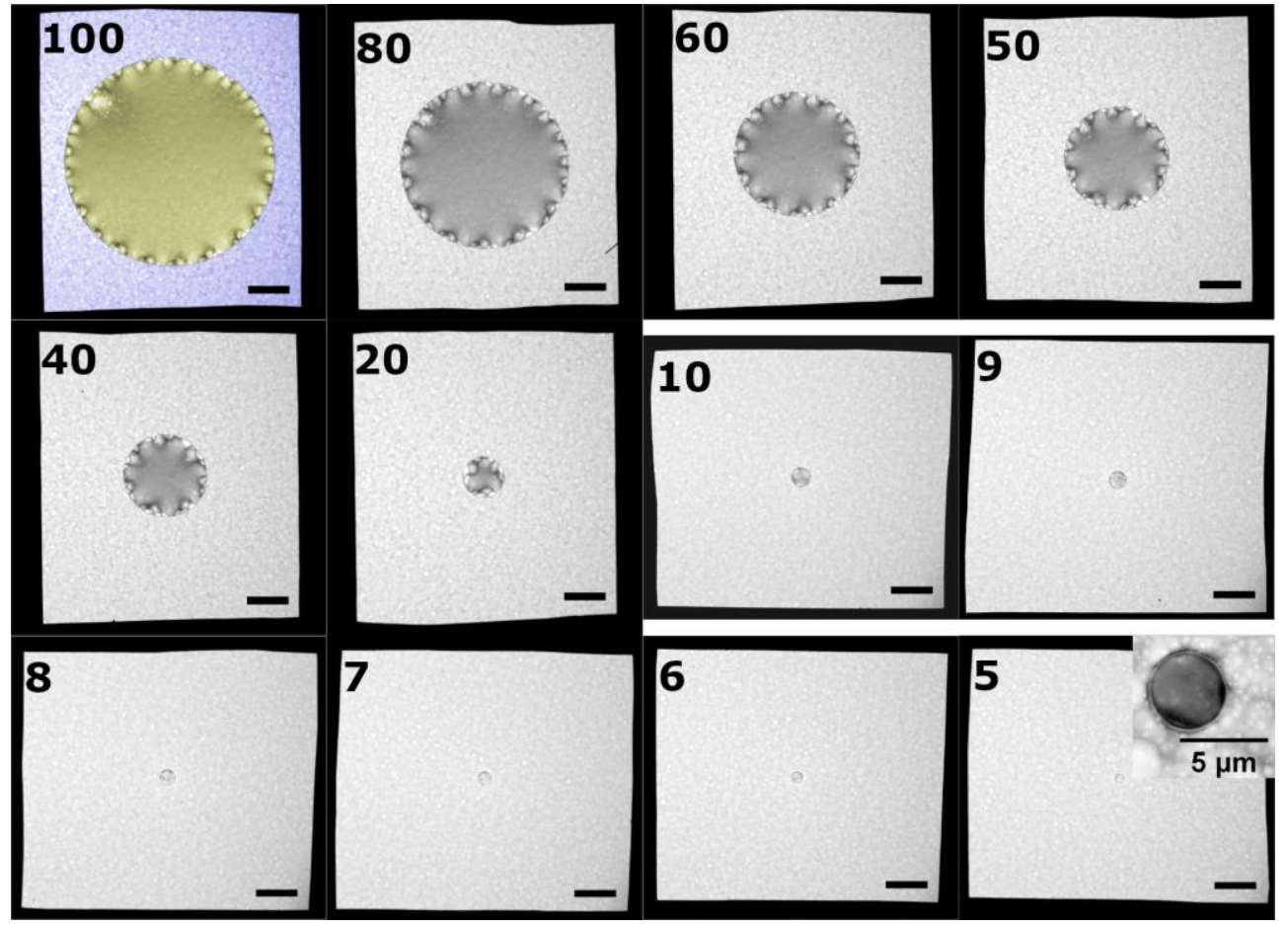

FIG. 4. Scanning transmission electron microscopy (STEM) images of the different circles investigated by Raman spectroscopy, main manuscript Fig. 3. The inner circle is the non-exposed silicon crystal (highlighted in yellow) called tympanum, while the lighter region is the implanted part (overlaid as blue color) dubbed tensioner. Scale bars on the STEM images represents $20 \mu \mathrm{m}$ and the top-left numbers correspond to the tympanum diameter.

\section{BI-MATERIAL BENDING THEORY}

The bilayer is shown in Fig. 5. The thicknesses of the substrate and film are $t_{s}$ and $t_{f}$, natural lengths of $L_{s}$ and $L_{f}$, and the misfit strain is $\Delta$. Note that in our case of an a-Si film produced by intermediate density gaseous species implants on c-Si we have negative $\Delta$ i.e. the film is shorter than the substrate. We approximate the shape of the bilayer to the arc of a circle (and we expect this to be an accurate approximation if the angle subtended, $\theta$, is small). The radius of curvature of the contact surface at $z=0$ is $R$.
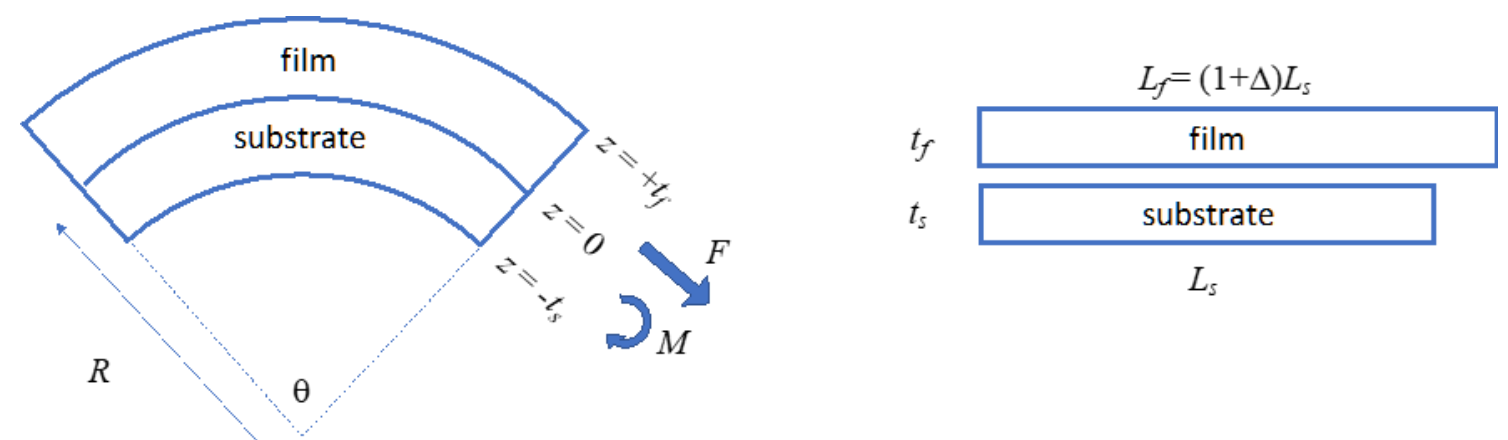

FIG. 5. A bent bilayer material. 
The strains in the substrate and film are

$$
\begin{aligned}
& \epsilon_{s}(z)=\frac{(R+z) \theta-L_{s}}{L_{s}}=\frac{z-z_{s}}{R_{s}}, \\
& \epsilon_{f}(z)=\frac{(R+z) \theta-L_{f}}{L_{f}}=\frac{z-z_{f}}{R_{f}},
\end{aligned}
$$

where

$$
\begin{gathered}
R_{s}=L_{s} / \theta, \\
z_{s}=R_{s}-R, \\
R_{f}=L_{f} / \theta, \\
z_{f}=R_{f}-R .
\end{gathered}
$$

$z_{s}$ and $z_{f}$ define the positions of the neutral surfaces (that have zero strain) for each layer. $R_{s}$ and $R_{f}$ do not have obvious physical meaning - they are the radii for curvature that the separate layers would have if they were to be bent into arcs subtending $\theta$. Clearly $R_{S}$ and $R_{f}$ are both close to $R$ and therefore $z_{S}$ and $z_{f}$ are small compared with $R$. Note that

$$
R_{f} / R_{s}=L_{f} / L_{s}=1+\Delta
$$

and

$$
\frac{z_{f}}{z_{S}}=\frac{R_{f}-R}{R_{S}-R}=\frac{R_{f} / R_{S}-R / R_{S}}{1-R / R_{S}}=\frac{1+\Delta-R / R_{S}}{1-R / R_{S}}=\frac{\Delta-\delta}{-\delta}=1-\frac{\Delta}{\delta}
$$

where

$$
\delta=R / R_{S}-1=-z_{S} / R_{S}=\epsilon_{S}(0)
$$

is the strain of the substrate layer at the contact surface $\mathrm{z}=0$, i.e.

$$
R=(1+\delta) L_{s} / \theta
$$

and

$$
z_{s}=-\delta R_{S}
$$

[In the absence of strong external forces, we would expect that the neutral surfaces are in the middle of the respective layers. For positive $\Delta$ the substrate is extended at the contact surface, i.e. $\delta$ is positive, and so the neutral surface of the substrate at $z_{S}$ is negative, as expected. If $z_{f}$ is positive, then $z_{f} / z_{S}$ is negative and $\Delta>\delta$.]

The stresses are

$$
\begin{aligned}
& \sigma_{s}(z)=E_{s} \epsilon_{s}(z)=E_{s} \frac{z-z_{s}}{R_{s}} \\
& \sigma_{f}(z)=E_{f} \epsilon_{f}(z)=E_{f} \frac{z-z_{f}}{R_{f}}
\end{aligned}
$$

where Es and $E_{f}$ are the Young's moduli. There is a force per unit width at each end that must exactly oppose the externally applied force in equilibrium:

$$
\frac{F}{w}=\int \sigma d z=\frac{E_{s}}{R_{s}} \int_{-t_{s}}^{0}\left(z-z_{s}\right) d z+\frac{E_{f}}{R_{f}} \int_{0}^{t_{f}}\left(z-z_{f}\right) d z
$$




$$
\begin{gathered}
=\frac{E_{s}}{R_{s}}\left[\frac{z^{2}}{2}-z_{s} z\right]_{-t_{s}}^{0}+\frac{E_{f}}{R_{f}}\left[\frac{z^{2}}{2}-z_{f} z\right]_{0}^{t_{f}} \\
=\frac{E_{s} t_{s}}{R_{s}}\left(-\frac{t_{s}}{2}-z_{s}\right)+\frac{E_{f} t_{f}}{R_{f}}\left(\frac{t_{f}}{2}-z_{f}\right) \\
=E_{s} t_{s}\left[\frac{1}{2} \frac{t_{s}}{R_{s}}\left(-1+\frac{E_{f} t_{f}^{2} R_{s}}{E_{s} t_{s}^{2} R_{f}}\right)-\frac{z_{s}}{R_{s}}\left(1+\frac{E_{f} t_{f} R_{s} z_{f}}{E_{s} t_{s} R_{f} z_{s}}\right)\right]
\end{gathered}
$$

Note that since the round brackets are of order unity and the prefactors in front of the round brackets are of similar order there are no additional approximations to be made. Finally

$$
\begin{aligned}
\frac{F}{w}=E_{s} t_{s}\left[\frac{\theta}{2} \frac{t_{s}}{L_{s}}\right. & \left.\left(-1+\frac{r_{E} r_{t}^{2}}{1+\Delta}\right)+\delta\left(1+\frac{r_{E} r_{t}\left(1-\frac{\Delta}{\delta}\right)}{1+\Delta}\right)\right] \\
& =E_{S} t_{s}\left[\frac{\theta}{2} \frac{t_{s}}{L_{s}}\left(-1+\frac{r_{E} r_{t}^{2}}{1+\Delta}\right)+\delta\left(1+\frac{r_{E} r_{t}}{1+\Delta}\right)-\frac{r_{E} r_{t} \Delta}{1+\Delta}\right]
\end{aligned}
$$

(1)

where the ratios of the Young's moduli and layer thicknesses are

$$
\begin{aligned}
& r_{E}=\frac{E_{f}}{E_{s}}, \\
& r_{t}=\frac{t_{f}}{t_{s}} .
\end{aligned}
$$

Note that for positive strain the internal stress is positive inwards and the external force is positive outwards (tensile). There is also a bending moment per unit width at each end that must exactly oppose the externally applied moment in equilibrium:

$$
\begin{gathered}
\frac{M}{w}=\int \sigma z d z=\frac{E_{s}}{R_{s}} \int_{-t_{s}}^{0}\left(z-z_{s}\right) z d z+\frac{E_{f}}{R_{f}} \int_{0}^{t_{f}}\left(z-z_{f}\right) z d z \\
=\frac{E_{s}}{R_{s}}\left[\frac{z^{3}}{3}-z_{s} \frac{z^{2}}{2}\right]_{-t_{s}}^{0}+\frac{E_{f}}{R_{f}}\left[\frac{z^{3}}{3}-z_{f} \frac{z^{2}}{2}\right]_{0}^{t_{f}} \\
=\frac{E_{s} t_{s}^{2}}{R_{S}}\left(\frac{t_{s}}{3}+\frac{z_{s}}{2}\right)+\frac{E_{f} t_{f}^{2}}{R_{f}}\left(\frac{t_{f}}{3}-\frac{z_{f}}{2}\right) \\
=E_{s} t_{s}^{2}\left[\frac{1}{3} \frac{t_{s}}{R_{s}}\left(1+\frac{E_{f} t_{f}^{3} R_{s}}{E_{s} t_{s}^{3} R_{f}}\right)+\frac{z_{s}}{2 R_{s}}\left(1-\frac{E_{f} t_{f}^{2} R_{s} z_{f}}{E_{s} t_{s}^{2} R_{f} z_{s}}\right)\right]
\end{gathered}
$$

Finally

$$
\begin{aligned}
\frac{M}{w}=E_{s} t_{s}^{2}\left[\frac{\theta}{3} \frac{t_{s}}{L_{s}}\right. & \left.\left(1+\frac{r_{E} r_{t}^{3}}{1+\Delta}\right)-\frac{\delta}{2}\left(1-\frac{r_{E} r_{t}^{2}\left(1-\frac{\Delta}{\delta}\right)}{1+\Delta}\right)\right] \\
& =E_{s} t_{s}^{2}\left[\frac{\theta}{3} \frac{t_{s}}{L_{s}}\left(1+\frac{r_{E} r_{t}^{3}}{1+\Delta}\right)-\frac{\delta}{2}\left(1-\frac{r_{E} r_{t}^{2}}{1+\Delta}\right)-\frac{r_{E} r_{t}^{2} \Delta}{1+\Delta}\right]
\end{aligned}
$$

(2) 
As before there are no additional approximations to be made. Note that for positive strain gradient in the positive z-direction the outer convex surface is under more tension than the inner concave surface and so the internal bending moment is positive anti-clockwise, so the external torque is positive clockwise.

In principle these equations can now be rearranged to obtain $\theta$ and $\delta$ (which describe the bending and stretching response of the bilayer) in terms of the externally applied force and torque, $F, M$, the material parameters $E_{s, f}$, the geometry variables $w, t_{s, f}$ and the two other parameters that define the natural lengths of the constituent layers, $L_{s}$ and the misfit strain $\Delta$.

For a free-standing cantilever, $F=M=0$, then eliminating $\delta$ from (1) and (2) and rearranging for $\theta$

$$
\begin{aligned}
& \theta=\frac{6 L_{s}}{t_{s}} \frac{r_{E} r_{t}\left(1+r_{t}\right) \Delta(1+\Delta)}{r_{E}^{2} r_{t}^{4}+r_{E} r_{t}^{2}\left(4 / r_{t}+6+4 r_{t}\right)(1+\Delta)+(1+\Delta)^{2}} \\
& =\frac{6 L_{s}}{t} \frac{\left(r_{t}+2+r_{t}^{-1}\right) \Delta(1+\Delta)}{r_{E} r_{t}^{2}+\left(4 / r_{t}+6+4 r_{t}\right)(1+\Delta)+r_{E}^{-1} r_{t}^{-2}(1+\Delta)^{2}}
\end{aligned}
$$

In the last step we divided top and bottom by $r_{E} r_{t}^{2}$ and used

$$
1+r_{t}=\left(1+t_{f} / t_{s}\right) \frac{t_{s}+t_{f}}{t}=\frac{\left(t_{s}+t_{f}\right)^{2}}{t_{s} t}=\frac{t_{f}}{t} \frac{t_{s}^{2}+2 t_{f} t_{s}+t_{f}^{2}}{t_{s} t_{f}}=\frac{r_{t} t_{s}}{t}\left(1 / r_{t}+2+r_{t}\right)
$$

If the misfit strain, $\Delta$, is small, then

$$
\theta=\frac{6 L_{s}}{t} \frac{2+\left(r_{t}+r_{t}^{-1}\right)}{6+4\left(r_{t}+r_{t}^{-1}\right)+\left(r_{E} r_{t}^{2}+r_{E}^{-1} r_{t}^{-2}\right)} \Delta
$$

which is simply proportional to $\Delta$. For negative misfit strain we have negative angle, obviously. Having obtained $\theta$ we can obtain the radius of curvature from $R_{S}=L_{S} / \theta$, and if all strains are small and the layers are thin then $R_{S} \approx R \approx R_{f}$ so if $\kappa=1 / R$ then

$$
\kappa=\frac{6}{t} \frac{2+\left(r_{t}+r_{t}^{-1}\right)}{6+4\left(r_{t}+r_{t}^{-1}\right)+\left(r_{E} r_{t}^{2}+r_{E}^{-1} r_{t}^{-2}\right)} \Delta
$$

This is identical to the famous Timoshenko formula for a bimetallic strip if $\Delta=\left(\alpha_{f}-\alpha_{s}\right) \delta T$. Since $x+x^{-1}=2+O\left(x^{2}\right)$ (i.e. no linear terms), $r_{t}$ or $r_{E}$ must be very far from unity for the following approximation to be invalid:

$$
\kappa \approx \frac{3 \Delta}{2 t}
$$

For very large $r_{t}$

$$
\kappa=\frac{6}{t} \frac{2+r_{t}}{6+4 r_{t}+r_{E} r_{t}^{2}} \Delta
$$

which decreases as $r_{t}$ increases. For very small $r_{t}$ the last result is the same with $r_{t}$ replaced by $1 / r_{t}$. 


\section{REFERENCES}

[1] K. Brunner, G. Abstreiter, B. O. Kolbesen, and H. W. Meul, Strain at $\mathrm{Si} \mathrm{SiO}_{2}$ interfaces studied by micron-Raman spectroscopy, Appl. Surf. Sci. 39, 116 (1989).

[2] R. J. Jaccodine and W. A. Schlegel, Measurement of strains at $\mathrm{Si}_{-} \mathrm{SiO}_{2}$ interface, J. Appl. Phys. 37, 2429 (1966).

[3] G. Taraschi, A. J. Pitera, and E. A. Fitzgerald, Strained Si, SiGe, and Ge on-insulator: Review of wafer bonding fabrication techniques, Solid. State. Electron. 48, 1297 (2004).

[4] D. B. Williams, and C. B. Carter, Transmission electron microscopy: A textbook for materials science. (Springer Science \& Business Media, 1996). 